\title{
Study on the application of accountability nursing in cardiology department
}

\author{
Chenglei Qi*1, Fang Jiang ${ }^{2}$ \\ ${ }^{1}$ Gaoqing County People's Hospital, Zibo, China \\ ${ }^{2}$ Gaoqing County People's Hospital, Zibo, China
}

\begin{abstract}
With the progress of medical technology, the treatment ability of central vascular diseases in hospitals is getting stronger and stronger, which improves patients' confidence in the treatment of diseases. In the department of cardiovascular medicine, in order to improve the effect of treatment and improve patients' satisfaction with medical services, bedside responsibility system nursing can be adopted for patients during the treatment process, which can effectively improve the quality and effect of nursing in the department of cardiovascular medicine and speed up the recovery of patients. This article narrated what is department of cardiovascular medicine, still elaborated the nursing method of bedside responsibility system in department of cardiovascular medicine and application effect.
\end{abstract}

\section{Introduction}

Due to the complexity and high risk of cardiology, problems in clinical nursing are easy to occur, which seriously affect the life and health of nursing staff. Through the system of accountability nursing management, specific responsibilities are assigned to individuals to ensure a clear division of labor within the team, so that each nursing staff clearly define their own responsibilities, improve the initiative of nursing, to provide nursing staff with high-quality, round-the-clock services. In the responsibility system nursing process, the main is to meet the needs of the patient's physiological and psychological aspects, at the same time to strengthen the training of nursing staff, let they can according to the actual situation of the patients recover for personalized nursing plan, in accordance with the whole nursing program at the same time, the related data of the patients with accurate records, fill in nursing documents for subsequent doctor-patient disputes to provide important reference basis. Rehabilitation guidance and health education can help relieve the suffering of patients, improve the level of basic care, and ensure that the overall level of nursing quality is significantly enhanced

With the advancement of medical system reform, the application of bedside accountability nursing in the department of cardiovascular Medicine in hospitals can not only effectively improve patients' satisfaction with nursing services, promote the establishment of good nurse-patient relationship, but also improve the safety and effect of treatment, and promote the recovery of patients. Bedside accountability nursing has high application value in cardiovascular internal medicine nursing, on the one hand, the application of bedside accountability nursing is not only

Improved patients' confidence in hospital treatment, improved patients' compliance; On the other hand, it is conducive to improving patients' satisfaction with medical services, thus reducing the incidence of medical disputes and improving the social influence of hospitals. In the bed of the responsibility system nursing application, need nurses have a strong sense of responsibility and the risk consciousness, in the cardiovascular internal medicine, strengthen the construction of the responsibility system nursing bed system, strengthen the training of nursing staff in a professional capacity, can improve the level of responsibility system nursing bed, promote the sustainable development of responsibility system nursing bed.[1]

\section{Department of Cardiovascular Medicine}

The department of cardiovascular Medicine is an important department of the hospital, which undertakes important treatment tasks in the hospital. This department is committed to the research and prevention of cardiovascular diseases. The types of cardiovascular diseases are diverse and the causes are complex. In the department of cardiovascular medicine, it is necessary to diagnose the diseases for patients first, and then make reasonable treatment plans for patients according to their actual conditions, so as to improve the therapeutic effect. The diseases treated by cardiovascular medicine include hypertension, pericarditis, myocardial infarction, atrial flutter, arrhythmia, angina pectoris, alcoholic cardiomyopathy, coronary heart disease, morning sickness, myocardial bridge, cardiomyopathy, heart failure,

\footnotetext{
*Corresponding author: 530113401@qq.com
} 
pericardial effusion, sudden death and other diseases. The doctor needs to pass strict examination before making diagnosis for the patient. In recent years, great progress has been made in the research of cardiovascular disease treatment in China, and the level of cardiovascular disease treatment is rising day by day. In the treatment of cardiovascular diseases, strengthening the application of nursing can effectively improve the quality of treatment and promote the benign development of cardiovascular medicine.[2]

\section{Bedside accountability nursing in cardiovascular medicine}

In recent years, in the medical service to carry out the "people-oriented" concept, in the cardiovascular internal medicine nursing, strengthen the responsibility system nursing bed, adhere to the "safe, continuous and balanced, level, responsibility" principle of the responsibility system nursing bed, deepen the "people-oriented" concept of medical services, to improve the nursing quality and level of cardiovascular internal medicine, improve patients satisfaction of nursing. In cardiovascular medicine, bedside responsibility system nursing methods mainly reflected in the following aspects.

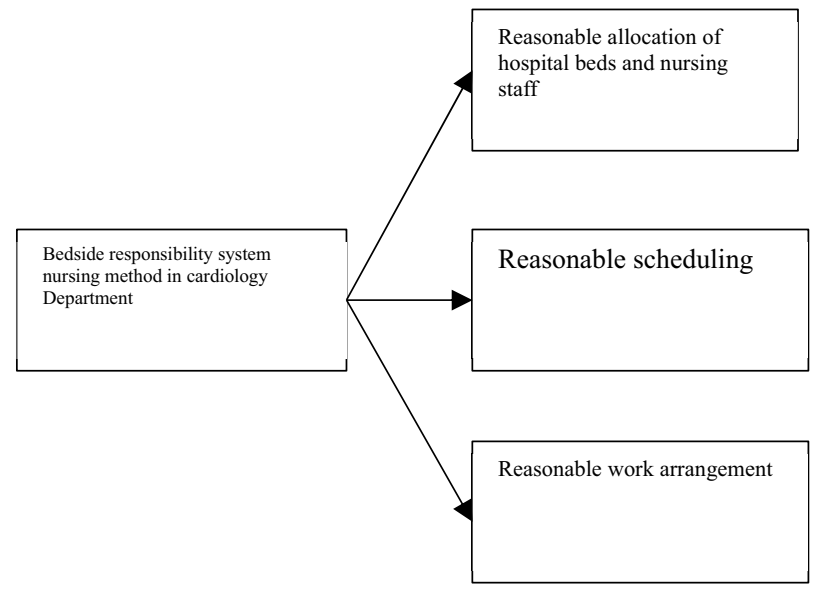

Figure 1. Bedside responsibility system nursing method in cardiology Department

\subsection{Reasonable allocation of hospital beds and nursing staff}

In recent years, the incidence of cardiovascular diseases is getting higher and higher. In the process of arranging hospital beds and nursing staff, the principle of equal distribution should be adhered to to improve the rationality of the arrangement. According to the specific situation of the department, the beds were divided into groups with the same number of beds in each group, and then one nurse was assigned to participate in bedside responsible nursing for each group of beds. In the process of distribution, need to pay attention to, participate in bedside responsibility nursing personnel, should not be in the night shift nursing work, on the one hand, improve the humanization of nursing work, on the other hand, improve the rationality of nursing work, improve the effect of nursing. In addition, the department according to their own actual situation, reasonable arrangement of bedside responsibility nursing staff number, improve the quality of bedside responsibility nursing work.[3]

\subsection{Reasonable scheduling}

The scheduling of bedside responsibility system nursing work should also be divided into 3 shifts to strengthen the connection between nursing staff and improve the quality of nursing. In the daytime, morning and afternoon shift scheduling, the department needs to reasonably arrange the number of nursing staff participating in bed care. Usually, it is most appropriate to arrange 2-3 bed nursing staff. In addition, medication administration care needs to be arranged for each group to improve the quality of care. According to the specific situation of the department, 1 nurse in the main class is arranged. In the night bed nursing, 2 nurses are assigned to be on duty nursing.[4]

\subsection{Work arrangement}

Bedside responsibility nursing staff nursing work is more complex, not only need to strengthen the observation of the patient's condition, but also need to combine with the actual situation of the patient, for the patient to develop a reasonable nursing plan, improve the quality of nursing, so as to promote the rehabilitation of patients. Nurses need to strengthen their understanding of the nature and content of their work to ensure the smooth completion of nursing work.

\section{Effect of bedside accountability nursing in cardiovascular medicine}

After the implementation of accountability management mode in cardiology nursing, dedicated nurses and patients with positioning management will have a longer time to communicate and care, which is conducive to the "doubleheart" management mode. One "heart" is the treatment and care of patients by cardiology nurses, while the other is the cardiology nurses pay more attention to patients' psychological problems. Patients gave higher evaluation on nurses' nursing attitude, nursing level, specialty ability, communication ability and health education, and patients' overall satisfaction was greatly improved, and they trusted nurses more. After the implementation of this mode, the responsible nurses can find the problems in time, analyze the problems, and come up with solutions quickly. When the problem is serious, nurses can communicate with the attending doctor to solve the problem as soon as possible, which can fill the gaps in clinical practice, strengthen the communication between doctors and nurses, and increase their cooperation and tacit understanding. Fundamentally, the overall optimization of nursing services, so that patients get better care and treatment.[5]

After implement the responsibility system for management in cardiology nursing, basic nursing and special nursing and nurse to the patient's propaganda education more time and energy into positive, make the relations between the nurses and patients more close to peace, and people are getting the thoughtful service, to the 
nurse's trust and satisfaction are improved significantly and at higher levels.

After the implementation of accountability management mode in cardiology nursing, nurses will have a longer time to contact with patients in their work, increase the active participation of junior nurses in patient care, and improve the quality of basic nursing. Senior nurses participate in patient management, pay more attention to the accumulation of work experience of critically ill patients, improve the overall care of critically ill patients, share resources, improve the critical thinking of nurses and emergency capacity. This management mode strengthened the cooperation, democracy, freedom, relaxed, harmonious working environment; The nursing process of each link and level is reduced, and the nursing service is refined. The relationship between the manager and the patient is closer, and the clinical nursing situation can be understood in real time. The work enthusiasm of nurses is greatly aroused, and the theoretical knowledge and operational skills of nurses are promoted to be further studied, so as to master the treatment of daily nursing and emergency from the overall perspective. You can make better use of your strengths to optimize team efficiency. Nurses are responsible for patients, their responsibilities are implemented to people, responsibilities are clear, work details are clear, reflects all patient-centered, everything for the sake of patients nursing philosophy, enhance the sense of responsibility of nurses, exercise comprehensive nursing ability and nursing quality. Responsibility, meanwhile, nurses and doctors together rounds visit patients, doctors and nurses more convenient comprehensive method, and familiar with treatment and nursing can largely improve the nurse work ability, enhance the consciousness of nurses service patients, in the play to their hard work at the same time, the value of increased nurses of self satisfaction.[6]

The overall quality of basic and specialized nursing has been improved obviously after the system of accountability management has been implemented in cardiology nursing. In addition to performing basic daily duties, the responsible nurse also pays attention to the physical and mental health of patients in the department of cardiology. While providing good basic and specialized nursing services for patients, patients should also be often talked with, in order to grasp the psychological status and living habits of patients, establish trust with patients, fully and deeply promote humanized nursing, promote the communication and trust between patients and medical staff. This management mode enables nurses to devote more time and energy to caring for patients and ensures the quality of nursing. At the same time, problems can be found in a timely manner, timely evaluation of the patient's physical condition and recovery; as a whole, it improves the connotation of nurses' work and plays a positive role in promoting the progress of nursing industry.

\section{Conclusion}

To sum up, in the study of cardiovascular medical nursing work found that strengthening the application of bedside accountability nursing, not only can effectively improve the quality of nursing, but also can improve the nursing level of nursing staff, promote the sustainable development of nursing services. Bedside responsibility system nursing fully reflects the concept of "peopleoriented" medical service, has higher clinical promotion value. In the process of responsible nursing team management, the specific responsibilities of the team must be deeply analyzed. Responsibility system nursing group mainly includes the disinfection quality team, drug quality management team, basic nursing quality inspection team, technology assessment team, the critically ill patient care quality inspection team, nursing documents quality inspection team, ward management quality inspection team, health education management standards group and nursing safety management team and other functions of different groups, the functional groups have different division of labor. The quality of weekly disinfection isolation shall be comprehensively recorded and reported, the risk of infection factors and weak links in the monitoring process shall be detailed, and improvement strategies shall be proposed.

The first-aid medical device inspection team must develop a complete first-aid medical device quality management standard, including a comprehensive inspection of oxygen device, sputum aspirator and other devices, and a comprehensive inspection of first-aid medical device quality every month to summarize relevant experience.

The ward management quality inspection team inspects the ward environment and nursing service quality, proposes improvement measures for existing problems and weak links, reports timely, and improves the communication management skills of health education. Regular implementation of health education inspection standards, to ensure that the quality of health education standard rate of more than $90 \%$. Strictly abide by the working time, stick to the post, can't leave the post without permission. Conscientiously implement the rules and regulations, strengthen the safety of the treatment and nursing of patients, timely observation of the patient's condition changes.

The symptoms of mutual buck-passing of responsibilities enable nursing staff to participate in nursing work more actively and effectively improve the overall quality and level of cardiology nursing. In addition, when the responsibility nursing team management is carried out, patients must be the center, reasonable analysis of all the needs of patients, and formulate corresponding solutions to help patients significantly improve the quality of life, so that patients feel more comfortable, so that more actively cooperate with the treatment. In cardiology nursing at the same time, the team leader must be to find and solve problems in time, the analysis of the problems properly, make the right intervention and guidance to ensure that, in the development of nursing work effectively in the process of care for, to improve the level of their own care, improve their professional ability and professional quality. By changing the original nursing service, the system of responsible nursing management can also enhance the comfort of patients, ensure that the nursing satisfaction of patients is greatly improved, strengthen the behavioral 
restraint and management of nursing staff, strengthen the confidence of patients and their families to overcome the disease, and ensure that each team member can do what he or she does. The nursing management mode of responsibility breaks the traditional nursing mode that centers on the cure of disease. Instead, it pays more attention to the quality of life and dignity of patients. The satisfaction degree and nursing quality assessment of patients in the inquiry group are significantly higher than those in the comparison group.

To sum up, the responsibility system of nursing management can comprehensively improve the overall quality of cardiology nursing, strengthen supervision and management, and also clarify the responsibilities of nursing staff. Only by implementing the responsibility system and nursing management can the overall nursing level of cardiology be significantly improved and help patients recover as soon as possible.

\section{References}

1. Che Jinghua. Study on management mode of responsibility nursing team in cardiology department [J]. Medicine :00189-00190.

2. Jihaian. Applied research on the management of responsible group leader in cardiology nursing [J]. Journal of Integrated Chinese and Western Medicine for Cardiovascular Diseases, 2019, 007(027):76.

3. Zhang Ning. Application of nurse responsibility system in emergency room of circulatory medicine $[\mathrm{J}]$. Chinese Medical Guide, 2020, V. 18(06):385-386.

4. Yang Chunhong. Application research of high quality nursing Model in cardiovascular internal Medicine nursing [J]. China Practical Medicine, 2019.

5. Sun Shuyan. Study on application experience of continuous nursing Quality Improvement in cardiology nursing [J]. Health reading, 2019(26).

6. Li Hongjie. Research on application experience of continuous nursing Quality improvement in cardiology nursing $[\mathrm{J}]$. Journal of Integrated Chinese and Western Medicine cardiovascular Diseases (Electronic Edition), 2019, 7(12):9-9. 